\title{
ANESTHÉSIE ET PROCÉDURES CHIRURGICALES POUR L'IMPLANTATION DE RADIO ÉMETTEURS DANS LA CAVITÉ VENTRALE DE TRUITES COMMUNES ADULTES (SALMO TRUTTA)
}

\author{
C. GOSSET*, J. RIVES
}

UMR ECOBIOP INRA/UPPA « Écologie Comportementale et Biologie des populations de poissons " Station INRA d'Hydrobiologie de Saint-Pée-sur-Nivelle, 64310 Saint-Pée-surNivelle, France. *Courriel : gosset@st-pee.inra.fr

Reçu le 22 décembre 2003

Received December 22, 2003

Accepté le 8 juillet 2004

Accepted July 8, 2004

\section{RÉSUMÉ}

L'objectif de cette étude est de déterminer la meilleure technique d'implantation chirurgicale intra-abdominale de radioémetteurs chez la truite commune (Salmo trutta). Deux types d'anesthésie, chimique au clou de girofle et par l'électricité sont comparés. Deux types d'émetteurs, à antenne filaire externe et à antenne interne enroulée et deux emplacements de l'incision de la paroi abdominale, en avant et en arrière de la ceinture pelvienne, sont testés.

Le clou de girofle est un anesthésique satisfaisant mais l'anesthésie par l'électricité est immédiate, et ne semble pas occasionner de stress post-opératoire. Elle offre un meilleur confort d'intervention que l'anesthésie chimique et ne présente aucun danger pour le poisson et l'expérimentateur. Elle convient donc parfaitement pour l'insertion chirurgicale des émetteurs de radiopistage.

Si on ne recherche pas une distance d'émission maximale, l'émetteur à antenne interne est préférable à l'émetteur à antenne externe: il est plus stable à l'intérieur de l'abdomen et son expulsion semble moins fréquente. Toutefois, la perte d'émetteur est relativement fréquente dans les deux cas et doit être prise en compte lors de toute surveillance par radiopistage, d'une durée supérieure à 40 jours. L'emplacement de l'incision n'a aucune influence sur la survie, le risque d'expulsion, la cicatrisation et le positionnement des marques dans l'abdomen.

Mots-clés: Anesthésie, clou de girofle, électricité, émetteur radio, marquage chirurgical.

\section{ANESTHESIA AND SURGERY PROCEDURES FOR IMPLANTING RADIO TRANSMITTERS INTO THE BODY CAVITY OF ADULT BROWN TROUT (SALMO TRUTTA)}

\begin{abstract}
The aim of this study was to determine the best intraperitoneal surgical tagging technique for brown trout (Salmo trutta) by radiotransmitter. Two types of anaesthesia, using either clove oil or electricity were compared. Two types of transmitters, with external trailing antenna or internal coil antenna, and two incision zones, anterior and posterior to the pelvic girdle, were tested.
\end{abstract}


Clove oil was a satisfactory anaesthetic but anaesthesia by electricity was immediate, and did not seem to cause post operational stress. It offered a better ease of intervention than chemical anaesthesia and presented no risks for fish and experimenter. It is thus perfectly suitable for the insertion of radiotracking transmitters.

If no maximum field range is needed, the internal antenna transmitter is preferable to the external antenna: it is more stable inside the abdomen and its expulsion seems less frequent. However, the loss of transmitter is relatively frequent in either case and must be taken into account during any monitoring by radiotracking, for duration longer than 40 days. In addition it does not seem that the location of the incision has an influence on survival, risk of expulsion, healing and situation of tags inside the abdomen.

Key-words: Anaesthesia, clove oil, electricity, radio transmitter, surgical tagging.

\section{INTRODUCTION}

Il est généralement admis, à l'heure actuelle, que la meilleure méthode de marquage par émetteur radio de la plupart des espèces de poissons pour des suivis de longue durée est l'implantation intra-péritonéale (MARINE RESEARCH INSTITUTE, 1999). La pratique de ce type d'implantation nécessite une immobilisation parfaite et donc une anesthésie profonde du poisson. Le choix de l'anesthésique et de la technique opératoire revêtent donc une grande importance car ils conditionnent, par leurs post effets, la capacité de récupération de l'animal.

II existe de nombreuses méthodes d'anesthésie que l'on peut classer en trois catégories principales (VETOFISH, 2002) :

- par immersion (benzocaïne, tricaïne (MS222), phénoxy-2-éthanol, clou de girofle...);

- par voie parentérale (alphaxalone, alphadolone, kétamine...), méthode peu utilisée pour le poisson ;

- non chimique (hypothermie, dioxyde de carbone, électronarcose).

L'efficacité de très nombreux anesthésiques chimiques - jusqu'à 16, (GILDERHUS et MARKING, 1987) - a été fréquemment comparée dans la littérature. Jusqu'à ces dernières années on a couramment utilisé le phénoxy-2-éthanol en France. Ce produit est actuellement fortement déconseillé en raison, notamment, de sa toxicité pour l'utilisateur (irritant pour la peau et dangereux pour les yeux) (DEROZIER, 1989) et des faibles différences existant entre les doses efficaces et les doses toxiques pour le poisson. Le méthane sulfonate de tricaïne (MS222) semble actuellement le plus utilisé en Amérique du Nord et est le seul anesthésiant chimique pleinement autorisé par les services vétérinaires pour les poissons. Le clou de girofle (85 à $90 \%$ d'eugénol) est de plus en plus utilisé et s'il n'est pas, à l'heure actuelle, pleinement autorisé en tant qu'anesthésiant, il n'a pas non plus été démontré qu'il était nocif pour l'homme. II est peu coûteux, agit à de faibles concentrations, est aussi efficace que le MS222 ou le phénoxy-2-éthanol, n'affecte pas la performance de nage (ANDERSON, SCOTT McKINLEY et COLAVECCHIA, 1997) et son facteur de sécurité (plage entre concentrations efficaces et concentrations toxiques) est important (CHANSEAU et al., 2002). Bien que le temps de récupération soit élevé, il représente, à l'heure actuelle, une bonne alternative à l'emploi des autres anesthésiants chimiques (MUNDAY et WILSON, 1997).

Les méthodes d'anesthésie non chimiques et l'anesthésie électrique en particulier ont été peu utilisées avec le poisson. Si l'anesthésie au dioxyde de carbone présente l'inconvénient majeur d'un temps de récupération très important, l'anesthésie électrique ou électronarcose en courant continu (galvanonarcose) présente pourtant des avantages 
certains pour des opérations qui ne nécessitent pas que l'on mette le poisson à sec: conservation intégrale de la respiration durant toute l'anesthésie et récupération immédiate du pouvoir de nage. Le principe en est simple: on soumet le poisson à un champ électrique de courant continu constant de manière à le maintenir en galvanonarcose (blocage des messages cérébraux vers les nerfs moteurs spinaux) (LAMARQUE, 1967 ; COWX et LAMARQUE, 1990). Ce champ électrique est obtenu lorsque l'on alimente en courant continu 2 électrodes couvrant les 2 extrémités d'une cuve à section rectangulaire (GOSSET, 1974). L'état du poisson est caractérisé par une immobilisation complète et une flaccidité musculaire qui peuvent être maintenues sans inconvénient pendant plusieurs dizaines de minutes.

Dans le cas d'un pistage de longue durée (supérieur à 1 mois) l'implantation chirurgicale d'un émetteur radio dans la cavité ventrale de la truite est la méthode la mieux adaptée: le poisson n'est pas gêné dans ses mouvements de nage et peut se nourrir normalement. Ce type de marquage ne semble pas affecter de manière significative l'évolution physiologique, le comportement ou les performances de nage de la truite arc-en-ciel (Onchorynchus mykiss) (MELLAS et HAYNES, 1985 ; LUCAS, 1989 ; STEVEN, LONG et PEARSONS, 1995 ; SWANBERG et GEIST, 1997 ; WAGNER et STEVENS, 1999) ou des juvéniles de saumon atlantique (Salmo salar) (MOORE, RUSSELL et POTTER, 1990 ; CONNORS et al., 2002), mais nous n'avons trouvé dans la bibliographie aucun travail de ce type concernant la truite commune (Salmo trutta). Deux types d'émetteur peuvent être utilisés suivant la taille du poisson et la distance d'émission nécessaire: à antenne filaire externe lorsqu'une distance maximale est requise, à antenne enroulée interne lorsque l'animal est de taille plus importante et que l'on peut accepter une distance d'émission plus faible. En effet, à autonomie et puissance consommée égales, un émetteur à antenne filaire est plus petit et sa distance de détection est plus importante (environ $40 \%$ ) qu'un émetteur à antenne enroulée. En ce qui concerne l'emplacement de l'incision, deux variantes principales de la méthode opératoire (en avant ou en arrière de la ceinture pelvienne) peuvent également être adoptées. Si ces différentes techniques ont déjà été expérimentées séparément et semblent avoir donné plus ou moins satisfaction, il n'existe, à notre connaissance, pas d'étude comparative exhaustive. WAGNER (WAGNER et STEVENS, 1999) a cependant montré qu'il n'existait pas de différence importante dans le comportement quand l'incision était pratiquée entre la ceinture pelvienne et les nageoires pectorales, soit sur le milieu de la surface ventrale (linea alba), soit sur le flanc droit à $1,5 \mathrm{~cm}$ de cette ligne. Par ailleurs, il semble intéressant de tester ces techniques sur la truite commune qui d'une part possède des caractères anatomiques différents de la truite arc-en-ciel (ceinture abdominale plus épaisse par exemple) et, d'autre part, présente un intérêt certain en matière de suivis migratoires (OVIDIO et PHILIPART, 2002). Pour des raisons évidentes de faisabilité les tests ont été effectués sur des truites de pisciculture, étant entendu qu'il existe également des différences anatomiques (masse musculaire en particulier) entre les poissons d'élevage et les poissons sauvages.

Le but de ce travail est de déterminer la technique opératoire la mieux adaptée à la truite commune adulte, en comparant les effets à court et moyen terme de deux types d'anesthésie (électrique et au clou de girofle), de deux emplacements de l'incision (en avant et en arrière de la ceinture pelvienne) et de deux types d'émetteur (à antenne interne enroulée et à antenne filaire externe).

\section{MATÉRIEL ET MÉTHODES}

L'expérimentation a été effectuée sur 60 truites communes de pisciculture disponibles, dont la taille était relativement hétérogène $(279 \mathrm{~g}<\mathrm{P}<700 \mathrm{~g}$; Pmoy $=428 \mathrm{~g}$ [SE $=12,1])$. On a constitué 6 lots de 10 poissons pris au hasard. Chaque truite a été pesée, mesurée et une identification individualisée a été réalisée à l'aide de 2 injections 
de bleu alcyan effectuées au dermo-jet, selon un emplacement codé. Ces opérations ont été pratiquées sous anesthésie, juste avant le marquage. Les poissons anesthésiés par l'électricité ont été pesés au préalable dans un récipient contenant de l'eau et mesurés à l'intérieur de la cuve de marquage à l'aide d'une règle plastique.

Pour l'anesthésie au clou de girofle il a été utilisé, pour 1 I d'eau, une solution de $0,04 \mathrm{ml}$ d'huile essentielle diluée dans $4 \mathrm{ml}$ d'alcool éthylique. Les différentes étapes de l'opération ont été caractérisées : T0 : mise du poisson dans la solution anesthésiante ; $\mathrm{T} 1$ : première perte d'équilibre qui se produit en moyenne $2 \mathrm{mn}$ ( $\mathrm{SE}=0,1 \mathrm{mn})$ après T0 ; T2 : ralentissement très important des battements operculaires qui se produit en moyenne $5,6 \mathrm{mn}(\mathrm{SE}=0,3 \mathrm{mn})$ après T0. On considère à ce moment que l'on a atteint l'anesthésie profonde (aucune réaction à la manipulation) nécessaire à l'intervention chirurgicale proprement dite; T3: début de l'opération (incision); T4: fin de l'opération; T5: reprise des battements operculaires; T6 : retour à la position d'équilibre. Pour effectuer l'intervention, on a utilisé une cuve en matière plastique (Figure 1) équipée d'un berceau de contention incliné. Le poisson anesthésié est placé dans ce berceau en forme de $\mathrm{V}$ et maintenu sur le dos de manière à ce que la partie supérieure de l'abdomen soit hors d'eau. A l'aide d'un tube souple introduit dans la bouche de la truite, on a maintenu une circulation forcée de l'eau de la cuve contenant l'anesthésiant par les ouïes, pendant toute la durée de l'opération (MARTY et SUMMERFIELD, 1986 ; MOORE, RUSSEL et POTTER, 1990 ; OVIDIO, 1999). Dès que l'opération a été terminée, les truites ont été mises en stabulation dans un bac oxygéné par un diffuseur d'air.

L'anesthésie à l'électricité a été effectuée dans la même cuve de forme parallélépipédique $(68 \times 21 \times 12 \mathrm{~cm})$ équipée de 2 plaques métalliques placées à chaque extrémité et faisant office d'électrodes. L'alimentation électrique est assurée par un générateur de courant continu. Le voltage inter-électrodes a été réglé à $40 \mathrm{~V}$ pour une eau de $240 \mu \mathrm{S} . \mathrm{cm}^{-1}$ de conductivité, ce qui, compte tenu de la déformation des lignes équipotentielles par le poisson, induit, pour une truite de $32 \mathrm{~cm}$ de longueur par exemple, une différence de potentiel tête-queue de 7,5 V environ. Dès qu'on le plonge dans la cuve la tête vers l'anode (pôle positif), le poisson est immédiatement immobilisé et ne réagit pas pendant l'opération chirurgicale, quelle que soit sa durée.

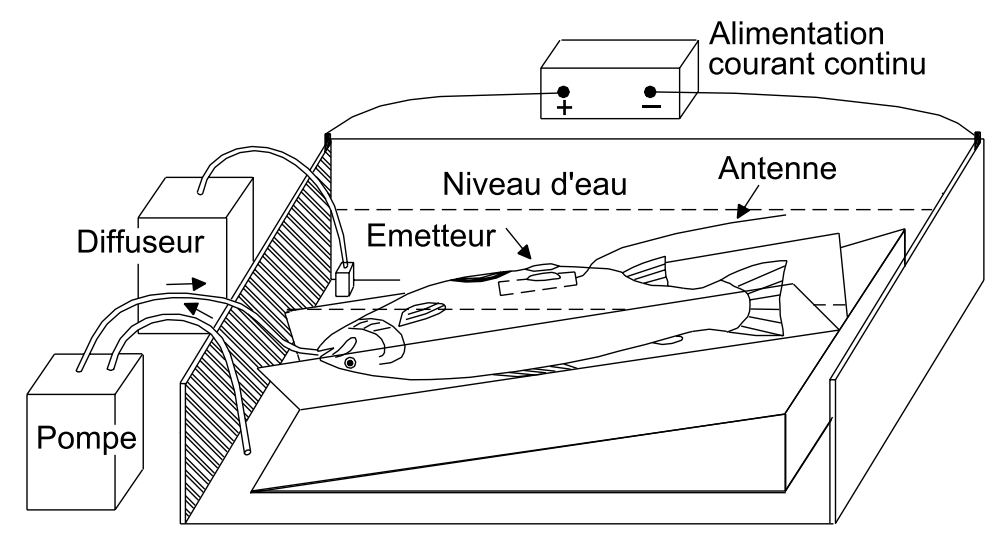

\section{Figure 1}

Dispositif d'anesthésie et de marquage chirurgical.

Figure 1

Device for anesthesia and surgical tagging. 
Avant chaque marquage, tous les instruments et l'émetteur ont été désinfectés à la bétadine. On a utilisé des émetteurs factices, moulés à partir d'une résine de type alimentaire inerte, de poids et de taille sensiblement équivalents aux émetteurs réels: $P=11 \mathrm{~g}, \mathrm{~L}=65 \mathrm{~mm}$, diam. $=12 \mathrm{~mm}$, pour les émetteurs à antenne enroulée et $P=8 \mathrm{~g}$, $\mathrm{L}=45 \mathrm{~mm}$, diam. $=11 \mathrm{~mm}$ pour les émetteurs à antenne filaire externe. L'introduction de l'émetteur dans la cavité abdominale a été effectuée de plusieurs manières :

- pour tous les émetteurs (antenne enroulée interne et antenne filaire externe), il a été pratiqué une incision de longueur juste suffisante pour permettre l'introduction de l'émetteur (1,5 à $2 \mathrm{~cm}$ ), soit entre la ceinture pelvienne et les nageoires pectorales (incision haute), soit entre la ceinture pelvienne et l'anus (incision basse). L'émetteur a été, dans tous les cas, positionné au niveau de la ceinture pelvienne de manière à ne pas modifier l'équilibre hydrostatique de l'animal. Lorsque l'incision est haute, on enfonce d'abord l'émetteur vers l'avant de la cavité pour ensuite le faire glisser au-dessus de la ceinture pelvienne ;

- pour les émetteurs à antenne filaire externe, l'antenne a été préalablement soudée à l'extrémité (côté chas) d'une aiguille à section triangulaire (3/8 cercle/00). Lorsque l'incision a été pratiquée en avant de la ceinture pelvienne on a introduit l'émetteur en commençant par l'aiguille que l'on a fait passer sous la ceinture pelvienne (Figure 2A) et fait ressortir entre celle-ci et l'anus (Figure 2B). Lorsque l'incision a été pratiquée en arrière de la ceinture pelvienne on a fait ressortir l'aiguille entre l'extrémité basse de l'incision et l'anus. II ne reste plus ensuite qu'à tirer sur l'aiguille pour entraîner l'antenne, positionner l'émetteur et se débarrasser de l'aiguille en sectionnant l'antenne au ras du chas Figure 2C). Le passage de l'aiguille doit être effectué avec soin, à l'aide d'une sonde cannelée, de manière à garder la pointe de l'aiguille dans la cavité ventrale pour ne pas occasionner de blessure interne. La protection de la pointe (ROSS et KLEINER, 1982) par un fourreau plastique ou le passage préalable d'une canule (STEVEN, LONG et PEARSON, 1995), qui ont pour inconvénient d'agrandir l'orifice de sortie de l'aiguille, ne nous ont pas paru nécessaires.

Les sutures ont été effectuées par 2 ou 3 points dits en « $U$ », à l'aide de fil «ETHICON PDS II 2-0 » semi-résorbable et d'une aiguille courbe 3/8T de $24 \mathrm{~mm}$. Une pommade grasse cicatrisante et non corticoïde (Fucidine $2 \%$ ) a ensuite été appliquée sur les lèvres de la cicatrice. Enfin on a procédé à une injection intra musculaire d'antibiotique (Nuflor $300 \mathrm{mg} / \mathrm{l}-0,3 \mathrm{ml} / \mathrm{kg}$ ). L'eau de la cuve a été fréquemment changée et une oxygénation correcte a été maintenue à l'aide d'un diffuseur d'air. La température de l'eau a varié entre $12^{\circ} \mathrm{C}$ et $14^{\circ} \mathrm{C}$.

Six lots de 10 truites individualisées par les numéros codés de 0 à 9 ont donc été constitués :

- $A H$ et $A B$ : lots témoin (incision et suture sans introduction d'émetteur) anesthésiés au clou de girofle ; 5 poissons avec incision haute $(\mathrm{AH})$ et 5 poissons avec incision basse $(\mathrm{AB})$;

- BB : anesthésie au clou de girofle, émetteur à antenne filaire externe et incision basse ;

- $\mathrm{BH}$ : anesthésie au clou de girofle, émetteur à antenne filaire externe et incision haute ;

- CB : anesthésie au clou de girofle, émetteur à antenne enroulée interne et incision basse ;

- $\mathrm{CH}$ : anesthésie au clou de girofle, émetteur à antenne enroulée interne et incision haute ;

- DH : anesthésie électrique, émetteur à antenne enroulée interne et incision haute. 


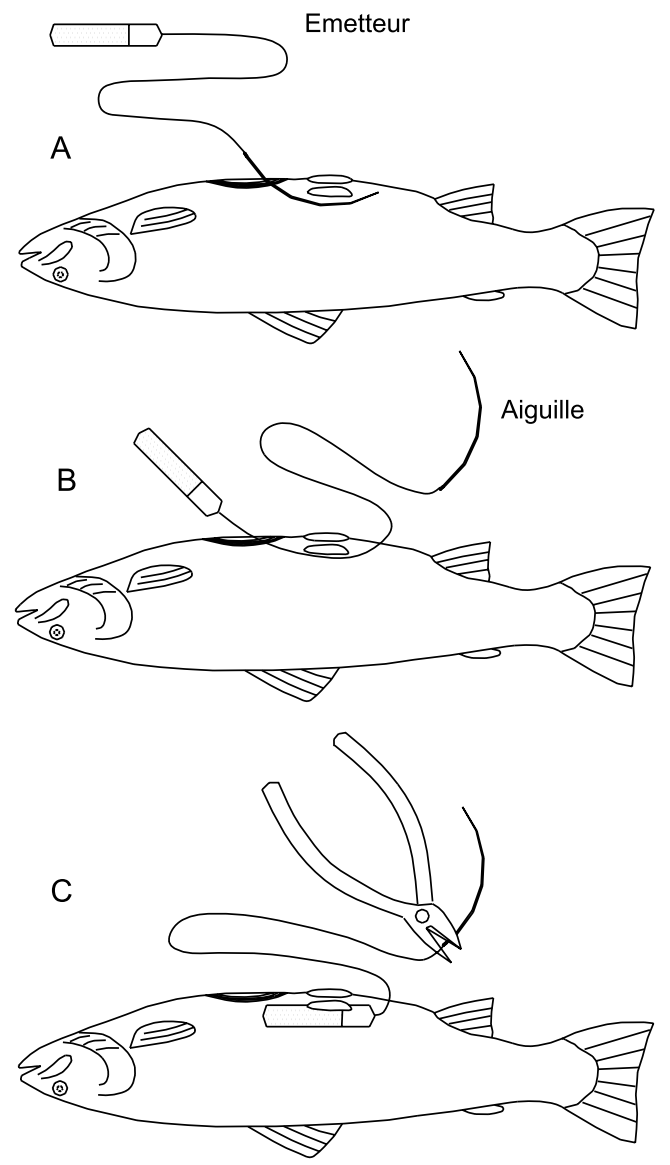

Figure 2

Insertion de l'émetteur radio à antenne filaire externe.

Figure 2

Implanting radio transmitter with whip antenna.

L'opération de marquage a eu lieu les 26 et 27 mars 2003. Les truites ont ensuite été stockées dans un bassin de pisciculture en terre de $20 \mathrm{~m}^{2}$ et nourries normalement à partir du $5^{\mathrm{e}}$ jour après le marquage. La température moyenne journalière a varié entre $10,4{ }^{\circ} \mathrm{C}$ et $21,8{ }^{\circ} \mathrm{C}$ au cours des 3 mois de stabulation. Le poids et la longueur de chaque poisson ainsi que l'état de la cicatrice, de la sortie antenne et l'état général sanitaire ont été contrôlés 5 fois : le 3 avril, le 10 avril, le 6 mai, le 28 mai et le 26 juin soit $1,2,6,9$ et 13 semaines après le marquage. Le 26 juin, toutes les truites ont été sacrifiées et disséquées. On a alors contrôlé l'état externe et interne de la cicatrice, relevé d'éventuelles inflammations ou nécroses des tissus et des organes, et observé la perte ou l'état et la situation de l'émetteur à l'intérieur de la cavité abdominale. La date de la perte éventuelle des émetteurs a été évaluée approximativement en observant le fond du bassin de stockage entre et lors des contrôles.

Les durées d'intervention, d'exposition à l'anesthésique et de récupération ainsi que la croissance ont été analysées par l'analyse de variance $\left(P_{0,05}\right)$. Le test de rapport de vraisemblance $\mathrm{G}$ (SOKAL et ROHLF, 2001) a été utilisé pour les comparaisons de mortalité, de perte de marques et de leur positionnement dans l'abdomen. Ce test, plus robuste que le test du Khi-deux, est donc préférable à celui-ci dans le cas de très petits effectifs. 


\section{RÉSULTATS}

\section{Anesthésie et opération de marquage}

La durée d'intervention (Tableau I) ne dépend pas du type d'anesthésie, du type d'émetteur ou du mode d'insertion (analyse de variance: $P=0,68$ ). Cette durée moyenne est de $8,2 \mathrm{mn}(\mathrm{SE}=0,2)$. Les durées d'exposition au clou de girofle sont donc également comparables $(P=0,34)$ ainsi que les diverses phases de la récupération. Une seule exception : la reprise du mouvement quasi-normal des opercules se produit plus rapidement avec le lot témoin « $A$ » qu'avec les autres lots $(P=0,03)$.

\section{Mortalité et croissance}

Une saprolégniose (champignon Saprolegnia) s'est développée dans le bassin de stockage et a probablement entraîné la quasi-totalité des mortalités (12). En effet, tous les poissons morts portaient des taches importantes sur tout le corps et en particulier sur la tête et la queue, alors que la cicatrisation paraissait satisfaisante. Aucune des 9 truites marquées mortes n'avait perdu l'émetteur. Pour stopper les mortalités (8 entre le 14/04 et le 27/04, 3 entre le 14/05 et le 21/05 et une le 19/06) des traitements au vert de malachite (5 à partir du 18/04) et au désogerme 3A (3 à partir du 28/04) ont été nécessaires. II n'y a aucune différence significative de mortalité entre les lots $(G=7,85$, df $=5, P=0,16)$ (Tableau II).

\section{Tableau I}

Durées moyennes ( \pm erreurstandard) d'intervention, d'anesthésie et de récupération, en relation avec le type d'anesthésique, d'émetteur et les techniques opératoires d'insertion : A : lot témoin ; BB : émetteur à antenne filaire et incision basse ; BH : émetteur à antenne filaire et incision haute ; $C B$ : émetteur à antenne enroulée et incision basse ; $\mathrm{CH}$ et $\mathrm{DH}$ : émetteur à antenne enroulée et incision haute.

(1) Moyenne pour les lots anesthésiés au clou de girofle.

(2) Lot anesthésié par l'électricité : le temps de l'anesthésie est celui de l'intervention (T4-T3).

\section{Table I}

Mean duration ( \pm standard error) for operation, anaesthesia and recovery in relation to the type of anaesthetic, transmitter, and surgical procedures; A: control fish; BB: transmitters with trailing antenna and backward of the pelvic girdle incision; $\mathrm{BH}$ : transmitters with trailing antenna and forward incision; CB: transmitters with coil antenna and backward incision; $\mathrm{CH}$ and DH: transmitters with coil antenna and forward incision.

(1) Mean for groups anaesthetised with clove oil.

(2) Fish anaesthetised with electricity: the duration of anaesthesia is that of the operation (T4-T3).

\begin{tabular}{|c|c|c|c|c|c|}
\hline Lots & $\begin{array}{c}\text { Durée } \\
\text { d'intervention } \\
\text { chirurgicale } \\
\text { (T4-T3) en mn }\end{array}$ & $\begin{array}{l}\text { Durée totale } \\
\text { d'exposition à } \\
\text { l'anesthésiant } \\
\text { (T4-T0) en mn }\end{array}$ & $\begin{array}{l}\text { Durée de fort } \\
\text { ralentissement } \\
\text { du mouvement } \\
\text { des opercules } \\
\text { (T5-T2) en mn }\end{array}$ & $\begin{array}{l}\text { Délai de reprise } \\
\text { du mouvement } \\
\text { des opercules - } \\
\text { fin d'intervention } \\
\text { (T5-T4) en mn }\end{array}$ & $\begin{array}{l}\text { Délai position } \\
\text { équilibre - fin } \\
\text { d'intervention } \\
\text { (T6-T4) en mn }\end{array}$ \\
\hline$A$ & $8,1 \pm 1,0$ & $15,8 \pm 1,4$ & $9,6 \pm 1,0$ & $0,6 \pm 0,1$ & $9,3 \pm 1,1$ \\
\hline BB & $7,8 \pm 0,4$ & $13,7 \pm 0,7$ & $10,3 \pm 0,7$ & $0,9 \pm 0,2$ & $9,7 \pm 0,6$ \\
\hline $\mathrm{BH}$ & $8,2 \pm 0,3$ & $13,7 \pm 0,4$ & $10,6 \pm 0,4$ & $1,7 \pm 0,3$ & $9,2 \pm 0,5$ \\
\hline CB & $8,5 \pm 0,3$ & $14,9 \pm 0,6$ & $10,5 \pm 0,4$ & $1,1 \pm 0,3$ & $11,1 \pm 0,7$ \\
\hline $\mathrm{CH}$ & $7,6 \pm 0,2$ & $14,6 \pm 0,5$ & $10,1 \pm 0,6$ & $1,4 \pm 0,3$ & $9,5 \pm 0,5$ \\
\hline Moy. (1) & $8,0 \pm 0,2$ & $14,6 \pm 0,4$ & $10,2 \pm 0,3$ & $1,2 \pm 0,2$ & $9,1 \pm 0,4$ \\
\hline $\mathrm{DH}^{(2)}$ & $8,9 \pm 0,6$ & & & & \\
\hline
\end{tabular}




\section{Tableau II}

Poids ( \pm erreur standard) et mortalité pour chaque lot. Croissance spécifique en $\%$ par jour ( \pm erreur standard) :

(1) de toutes les truites vivantes en fin d'expérimentation ;

(2) de toutes les truites vivantes ayant conservé l'émetteur en fin d'expérimentation.

\section{Table II}

Weight ( \pm standard error) and mortality for each group. Specific growth in \% per day ( \pm standard error):

(1) of all trout still alive at the end of experimentation;

(2) of all trout still alive having retained their transmitters at the end of experimentation.

\begin{tabular}{ccccc}
\hline Lots & $\begin{array}{c}\text { Poids moyen } \\
\text { au marquage }(\mathrm{g})\end{array}$ & Mortalité & $\begin{array}{c}\text { Croissance spécifique } \\
\text { après } 2 \text { sem. }{ }^{(1)}\end{array}$ & $\begin{array}{c}\text { Croissance spécifique } \\
\text { après } 3 \text { mois }\end{array}$ \\
\hline $\mathrm{A}$ & $363 \pm 31$ & 3 & $0,33 \pm 0,11$ & $0,39 \pm 0,04$ \\
$\mathrm{BB}$ & $365 \pm 12$ & 0 & $0,15 \pm 0,08$ & $0,36 \pm 0,06$ \\
$\mathrm{BH}$ & $364 \pm 11$ & 2 & $0,16 \pm 0,09$ & $0,23 \pm 0,04$ \\
$\mathrm{CB}$ & $480 \pm 16$ & 2 & $0,14 \pm 0,10$ & $0,25 \pm 0,03$ \\
$\mathrm{CH}$ & $547 \pm 27$ & 1 & $0,06 \pm 0,06$ & $0,20 \pm 0,03$ \\
$\mathrm{DH}$ & $485 \pm 17$ & 4 & $0,17 \pm 0,06$ & $0,25 \pm 0,02$ \\
\hline
\end{tabular}

La croissance spécifique ( $\mathrm{Cs}=\frac{\log \mathrm{P} 2-\log \mathrm{P} 1}{\mathrm{~T} 2-\mathrm{T} 1}$, exprimée en $\%$ par jour) a été analysée 2 semaines après le marquage, sur toutes les truites vivantes en fin d'expérimentation et, à la fin de l'expérimentation, sur toutes les truites vivantes ayant conservé l'émetteur (Tableau II). Deux semaines après le marquage il n'apparaît pas de différence significative de la croissance spécifique entre tous les lots (analyse de variance : $P=0,53$ ) ou entre les lots effectivement marqués (analyse de variance : $P=0,91$ ). En fin d'expérimentation, il n'y a pas de différence significative de croissance entre les différents lots de poissons marqués (analyse de variance : $P=0,10)$ mais cette différence devient significative $(P=0,01)$ si on inclut le lot témoin « $A$ » dans l'analyse. Ceci revient à dire que la croissance sur 3 mois n'est pas significativement différente selon le type de marquage mais que la présence de la marque dans l'abdomen a ralenti la croissance.

\section{Perte d'émetteurs}

Onze truites au total sur les 41 survivantes marquées (près de $27 \%$ ) ont perdu l'émetteur : 10 entre le 6/05 et le 28/05 (soit entre 6 et 9 semaines après le marquage) et une entre le 28/05 et le 26/06 (soit entre 9 et 13 semaines après le marquage). La répartition de ces pertes par lot est donnée au tableau III. II n'existe pas de différence significative entre les lots $(G=3,85, \mathrm{df}=4, \mathrm{P}=0,42)$. Les pertes d'émetteurs à antenne externe se produisent presque toutes (6/7) par une plaie qui s'est ouverte à partir du trou de passage de l'antenne (agrandissement du trou). La dernière (lot $\mathrm{BH}$ ) a eu lieu par une plaie qui s'est formée dans la paroi abdominale, sur la partie inférieure du flanc droit, un peu en avant des pelviennes. En ce qui concerne les antennes enroulées, l'émetteur a, dans tous les cas, été expulsé par une plaie située dans la partie basse de l'abdomen, près de l'anus. Excepté le cas particulier de l'expulsion par le flanc, on constate que, quel que soit le type d'émetteur et d'incision, les pertes se sont produites en aval de la ceinture pelvienne, dans la partie basse du ventre. Les pertes de marques avec antenne 
externe sont plus importantes mais la différence n'est pas significative $(G=2,37, d f=1$, $P=0,12)$. L'emplacement de l'incision n'a aucune influence sur le nombre de pertes des émetteurs à antenne externe $(G=1,19, \mathrm{df}=1, \mathrm{P}=0,27)$ ou des émetteurs à antenne enroulée $(G=0,21, d f=1, P=0,64)$.

Le processus d'expulsion a été pour chaque cas pratiquement identique: tout d'abord apparition d'un gonflement à l'endroit de l'expulsion puis, très rapidement développement d'une inflammation et d'une nécrose avant l'ouverture d'une plaie et l'expulsion de l'émetteur qui a été retrouvé sur le fond du bassin. Quelle que soit la situation de la plaie, celle-ci s'est parfaitement cicatrisée et est devenue 3 à 4 semaines plus tard presque indécelable à l'œil nu.

Tableau III

Perte, situation et encapsulation des émetteurs à l'intérieur de la cavité abdominale, pour chaque lot, 3 mois après le marquage.

Table III

Loss, situation and encapsulation of the transmitters in the body cavity for each group, 3 months after tagging.

\begin{tabular}{|c|c|c|c|c|c|c|c|}
\hline \multirow{2}{*}{ Lots } & \multirow[b]{2}{*}{$\begin{array}{c}\text { Total } \\
\text { marques } \\
\text { implantées }\end{array}$} & \multirow[b]{2}{*}{$\begin{array}{l}\text { Marques } \\
\text { perdues }\end{array}$} & \multicolumn{4}{|c|}{$\begin{array}{l}\text { Marques gardées : position par } \\
\text { rapport à la ceinture pelvienne }\end{array}$} & \multirow[b]{2}{*}{$\begin{array}{c}\text { Marques } \\
\text { gardées } \\
\text { encapsulées }\end{array}$} \\
\hline & & & Arrière & Centré & Avant & Total & \\
\hline BB & 10 & 5 & 1 & 0 & 4 & 5 & 2 \\
\hline $\mathrm{BH}$ & 8 & 2 & 0 & 0 & 6 & 6 & 3 \\
\hline $\begin{array}{l}\text { Total ant. } \\
\text { externes }\end{array}$ & 18 & 7 & 1 & 0 & 10 & 11 & 5 \\
\hline CB & 8 & 1 & 0 & 3 & 4 & 7 & 2 \\
\hline $\mathrm{CH}$ & 9 & 2 & 2 & 1 & 4 & 7 & 4 \\
\hline $\mathrm{DH}$ & 6 & 1 & 0 & 3 & 2 & 5 & 3 \\
\hline $\begin{array}{l}\text { Total ant. } \\
\text { internes }\end{array}$ & 23 & 4 & 2 & 7 & 10 & 19 & 10 \\
\hline
\end{tabular}

\section{Cicatrisation}

Lors du contrôle du 10/04 soit 2 semaines après le marquage, $55 \%$ des plaies sont pratiquement refermées et ne présentent pas d'inflammation caractérisée. Les autres présentent une inflammation plus ou moins importante avec les lèvres de la plaie parfois légèrement écartées. Lors du contrôle du 6/05 (6 semaines après le marquage), toutes les plaies sont refermées et il ne subsiste qu'une inflammation mineure près de l'incision pour 2 individus. En revanche, 2 émetteurs sont déjà expulsés par le trou de l'antenne et des gonflements de l'abdomen apparaissent aux endroits (flanc, entre pelviennes et anus) où les émetteurs vont être plus tard expulsés (cf. paragraphe précédent). Le 28/05 ( 2 mois après le marquage), toutes les plaies opératoires sont pratiquement cicatrisées. Les plaies d'éjection de l'émetteur sont cicatrisées pour 6 individus. Pour 3 autres truites elles sont encore visibles et plus ou moins enflammées. Un gonflement important et une inflammation sont visibles sur la partie basse du flanc droit d'une truite, un peu en avant des pelviennes ; l'émetteur sera expulsé quelques jours plus tard à cet endroit. Lors du 
dernier contrôle du 26/06, soit 3 mois après le marquage, toutes les plaies, y compris les orifices de sortie des émetteurs, sont parfaitement cicatrisées, il apparaît simplement quelques inflammations mineures autour de quelques points de suture non résorbés.

\section{Situation de l'émetteur dans l'abdomen}

Trois mois après le marquage l'émetteur a le plus souvent changé de place dans la cavité ventrale (23 sur 30 ). La position centrale de la marque est mieux conservée avec l'antenne interne (7/19) qu'avec l'antenne externe $(0 / 11)(G=7,58, d f=1, P=0,006)$, et l'émetteur à antenne externe se situe plus souvent en avant (10/11 contre 10/19) $(G=5,20, d f=1, P=0,02)$. L'emplacement de l'incision n'a aucune influence sur la position de la marque $(G=0,08, d f=2, P=0,96)$. En revanche, si l'on considère que les marques perdues se sont d'abord déplacées vers l'arrière (7 pour l'antenne externe et 4 pour l'antenne interne), on observe également que l'émetteur à antenne interne est plus stable $(7 / 23$ contre $0 / 18)(G=9,21, d f=1, P=0,02)$ mais que le type d'antenne n'influe pas sur le sens du déplacement, puisque au total on a 14 déplacements vers l'arrière (respectivement 8 et 6 ) contre 20 vers l'avant (10 et 10) ce qui n'est pas significativement différent $(G=0,17, d f=1, P=0,68)$.

Tous les émetteurs sont plus ou moins enrobés de tissus : la moitié au moins sont fortement encapsulés dans un épais tissu cellulaire, les autres sont enrobés dans une membrane plus ou moins épaisse qui est parfois attachée ponctuellement aux intestins. Parfois ( 3 cas), les émetteurs sont passés sous les intestins et se trouvent pratiquement en contact avec le rein. Le taux d'encapsulation ne dépend ni du type d'émetteur $(G=0,14$, $d f=1, P=0,70)$, ni de l'emplacement de d'incision $(G=2,25$, $d f=1, P=0,13)$.

\section{DISCUSSION}

Lors de l'anesthésie au clou de girofle, le fort ralentissement du mouvement des opercules (T2) est un critère subjectif car il se fait d'une manière assez progressive : les battements deviennent irréguliers puis saccadés avec des soubresauts avant de devenir pratiquement imperceptibles. De même, le retour à la position d'équilibre (T6) se déroule en $1 \mathrm{mn}$ environ, avec une succession fréquente de redressements et de déséquilibres. Les durées moyennes d'anesthésie profonde $(8,2 \mathrm{mn})$ sont comparables avec celles que l'on trouve généralement dans la littérature, obtenues avec d'autres anesthésiants chimiques et d'autres espèces : 9,5 mn avec du MS-222 et 7,9 mn avec du 2-phénoxy-2-éthanol par exemple pour le tilapia (Oreochromis aureus) (THOREAU et BARAS, 1996). Malgré une durée moyenne d'exposition à l'anesthésiant relativement importante (14,6 mn), tous les poissons ont récupéré leur équilibre et leur pouvoir de nage et aucune mortalité postopératoire n'est intervenue dans les premières 48 heures. Avec l'anesthésie électrique, il n'y pas d'anesthésie préalable et, comme le poisson récupère immédiatement son pouvoir de nage dès qu'il n'est plus exposé au courant électrique, sa durée est réduite à la durée d'intervention $(9 \mathrm{mn})$. Par ailleurs, l'anesthésie électrique peut être prolongée sans danger: on peut donc dire que le confort d'intervention est meilleur comparativement à l'anesthésie au clou de girofle, avec laquelle il existe un risque d'anoxie si la ventilation des ouïes est mal assurée. LAMARQUE (LAMARQUE, 1967) déconseille l'emploi de tout autres types de courant électrique pour l'immobilisation du poisson, à cause des réactions nerveuses et musculaires dangereuses qu'ils peuvent induire (tétanies par exemple), même si MADDEN (MADDEN et HOUSTON, 1976) n'a pas constaté d'effets physiologiques avec un type de courant plus choquant (impulsions alternatives) et un voltage plus élevé. La galvanonarcose, pratiquée dans les conditions de cette expérimentation est donc très bien adaptée à ce type d'intervention. En revanche, elle ne permet pas la manipulation du poisson hors de l'eau, ce qui rend plus difficile la pesée ou certaines mensurations spécifiques précises. 
II est fort probable que les mortalités aient été provoquées essentiellement par la saprolégniose. En effet, dans une eau qui n'est pas de qualité irréprochable - malgré les traitements antifongiques et antimicrobiens appliqués régulièrement dans cette pisciculture, des mortalités par saprolégniose ont été constatées sur les truites en élevage, au cours de la même époque - le poisson manipulé (perte de mucus et d'écailles) est prédisposé à une telle infestation. Mais si les traitements appliqués ont stoppé l'évolution de la saprolégniose, on ne peut malheureusement affirmer qu'ils n'ont pas modifié une mortalité éventuelle imputable à l'insertion de l'émetteur (infection, blocage du transit intestinal, organes internes endommagés) maintes fois constatée (MARINE RESEARCH INSTITUTE, 1999). Bien que la prise de nourriture a semblé s'effectuer de façon normale, on observe un taux de croissance relativement faible ( 0,2 à $0,4 \%$ par jour) en comparaison de celui que l'on obtient généralement en pisciculture. II est probable que la saprolégniose est également responsable (sans que l'on puisse dire dans quelle mesure) de cette faible croissance. En outre, ce ralentissement de la croissance semble plus important sur les poissons marqués que sur les poissons non marqués après 2 semaines (différence non significative) et plus encore sur toute la durée de l'expérimentation (différence significative), ceci malgré une variabilité individuelle importante. Ce ralentissement de la croissance dû au marquage a déjà été signalé dans le cas de la truite arc-en-ciel (Onchorynchus mykiss) après 7 mois (LUCAS, 1989) ou après 47 jours (MARTIN, LONG et PEARSONS, 1995) et du juvénile de saumon (Salmo salar) après 28 jours (MOORE, RUSSEL et POTTER, 1990).

L'expulsion des émetteurs est relativement fréquente $(27 \%$ des marques ont été perdues). Les premières pertes n'ont pu, compte tenu de la mauvaise visibilité au fond du bassin, être situées avec précision dans le temps mais sont intervenues entre 40 et 62 jours après le marquage. Même si MARTIN (MARTIN, LONG et PEARSONS, 1995) n'a observé aucune perte de marque 47 jours après le marquage de truites arc-en-ciel, la plupart des auteurs font état de taux de perte parfois importants dans des délais plus ou moins longs : $9 \%$ chez la truite arc-en-ciel après 110 jours (LUCAS, 1989), $20 \%$ chez les juvéniles de saumon après 120 jours (MOORE, RUSSEL et POTTER, 1990), 59 \% chez la truite arc-en-ciel entre 42 et 175 jours après le marquage (CHISHOLM et HUBERT, 1985). En ce qui concerne d'autres espèces, BARAS (BARAS et JEANDRAIN, 1998) ne constate pas d'expulsion après 12 semaines chez l'anguille (Anguilla anguilla) mais, en revanche, des taux de pertes très importants sont observés chez le poisson chat (Ictalurus punctatus) : $71 \%$ des émetteurs ont été expulsés après 112 jours (SUMMERFELT et MOSIER, 1984) et $52 \%$ après 23 jours (MARTY et SUMMERFIELD, 1986). Toutes espèces réunies, $22 \%$ des études mentionnent un taux d'expulsion supérieur à $20 \%$ (MARINE RESEARCH INSTITUTE, 1999). Lors de notre expérimentation, l'expulsion des émetteurs semble être favorisée par la présence de l'orifice effectué pour le passage de l'antenne (7 sur 18) mais s'est aussi produite par une déchirure de la paroi abdominale lorsqu'il n'y avait pas d'antenne (4 sur 23). Si l'expulsion par la face ventrale est relativement courante, l'expulsion par la paroi latérale est plus rare mais a déjà été constatée. Elle serait due à la pression de l'extrémité de la marque sur la paroi ventrale, ce qui entraîne d'abord une nécrose locale puis la formation d'une plaie et l'ouverture d'un passage au travers des tissus (LUCAS, 1989). Des expulsions par la voie intestinale et l'anus ont également été observées chez le poisson chat. Le processus d'expulsion est alors le suivant : encapsulation de la marque par une membrane, adhésion de cette membrane aux intestins, inflammation locale, affaiblissement du tissu intestinal et enfin passage de l'émetteur dans l'intestin (SUMMERFELT et MOSIER, 1984 ; MARTY et SUMMERFELT, 1986). D'une manière générale, l'expulsion n'entraîne pas la mort du poisson. C'est ce qui a également été observé dans le cas de notre expérimentation, où l'expulsion ne semble pas avoir entraîné de mortalités puisque aucune des truites mortes n'avait perdu l'émetteur. En outre, la cicatrisation des plaies s'est toujours effectuée de manière quasiparfaite. 
Dans tous les cas et en particulier pour l'antenne externe, il semble que les émetteurs qui se sont déplacés en aval de la ceinture pelvienne aient eu tendance à être expulsés. Cela s'est produit souvent par le trou de l'antenne filaire qui ne peut se cicatriser compte tenu du frottement permanent de l'antenne sur l'orifice de sortie. Une légère inflammation et un agrandissement de ce trou étaient visibles, à la fin de l'expérimentation, sur les individus ayant conservé l'émetteur. En revanche cet orifice était parfaitement cicatrisé quand l'émetteur avait été éjecté. Même si la différence n'est pas significative, il semble que l'émetteur à antenne interne soit expulsé moins souvent que l'émetteur à antenne externe. En outre, il conserve mieux la position centrale d'équilibre et a une plus grande stabilité dans la cavité ventrale et ceci quel que soit l'emplacement de l'incision.

Bien que le bassin de stockage en terre $\left(20 \mathrm{~m}^{2}\right.$ et $1 \mathrm{~m}$ de profondeur d'eau) reflète davantage le milieu de vie des truites sauvages que l'aquarium ou le bac d'élevage, les résultats de cette mise au point méthodologique ne sont sans doute pas totalement transposables au milieu naturel. Même si plusieurs auteurs ont démontré que le comportement (compétition, dominance) est peu affecté par le marquage, il est probable que la forte densité d'individus a entraîné des réactions épisodiques d'agressivité (CONNORS et al., 2002) que l'on ne retrouve pas dans le milieu naturel et qui peuvent avoir une influence négative sur les post-effets du marquage (inflammation de la plaie et perte d'émetteur). Par ailleurs, la truite sauvage possède une ceinture abdominale plus épaisse que la truite d'élevage, ce qui peut permettre une meilleure rétention des marques, surtout dans le cas des émetteurs à antenne externe. En conséquence, on peut espérer que dans le milieu naturel la cicatrisation se fera dans de meilleures conditions et que le taux de pertes sera moins élevé mais ceci reste bien entendu à vérifier.

\section{CONCLUSION}

Le clou de girofle est un anesthésiant satisfaisant mais nécessite, comme tous les anesthésiants chimiques, une mise en œuvre préalable à l'intervention et un temps de récupération relativement important. L'anesthésie à l'électricité permet une immobilisation immédiate, complète et durable du poisson, qui récupère immédiatement son pouvoir de nage après l'opération. Elle offre un meilleur confort d'intervention que l'anesthésie chimique puisque l'opérateur n'est pas pressé par le temps. Son utilisation ne présente aucun danger, ni pour le poisson ni pour les expérimentateurs. L'anesthésie à l'électricité nécessite cependant un appareillage simple mais adapté. Elle convient parfaitement pour l'insertion chirurgicale des émetteurs de radiopistage.

Lorsqu'on ne désire pas une distance d'émission maximale nécessitant obligatoirement une antenne externe, l'utilisation d'émetteurs à antenne interne enroulée est préférable : les risques d'infection sont moins importants, il est plus stable à l'intérieur de l'abdomen et son expulsion semble moins fréquente. Toutefois, le taux de perte d'émetteur chez la truite commune d'élevage est relativement important. Même s'il est possible que la fréquence des pertes soit inférieure dans le cas de la truite sauvage en milieu naturel, cette possibilité de pertes de marques doit être prise en compte lors de toute surveillance par radiopistage, d'une durée supérieure à 40 jours. Par ailleurs, II ne semble pas que l'emplacement de l'incision ait une influence sur la survie, le risque d'expulsion ou la cicatrisation.

\section{REMERCIEMENTS}

Nous remercions les agents INRA de la pisciculture de Lees-Athas pour la fourniture des truites, le Lycée Agricole de Saint-Pée-sur-Nivelle pour la mise à disposition gracieuse d'un bassin d'élevage, Patrick Girard et Jean-Marie Blanc pour leurs précieux conseils ainsi que les correcteurs anonymes pour leurs corrections et suggestions. 


\section{BIBLIOGRAPHIE}

ANDERSON W.G., SCOTT McKINLEY R.S., COLAVECCHIA M., 1997. The use of clove oil as an anaesthesic for rainbow trout and its effects on swimming performance. North American Journal of Fisheries Management, 17, 301-307.

BARAS E., JEANDRAIN D., 1998. Evaluation of surgery procedures for tagging eel Anguilla anguilla (L.) with biotelemetry transmitters. Hydrobiologia, 371/372, 107-111.

CHANSEAU M., BOSC S., GALIAY E., OULES G., 2002. L'utilisation de l'huile de clou de girofle comme anesthésique pour les smolts de Saumon atlantique (Salmo salar L.) et comparaison de ses effets avec ceux du 2-phénoxyéthanol. Bull. Fr. Pêche Pisc., 365/366, 579-589.

CHISHOLM I.M., HUBERT W.A., 1985. Expulsion of dummy transmitters by rainbow trout. Transactions of the American Fisheries Society, 114, 766-767.

CONNORS K.B., SCRUTON D., BROWN J.A., McKINLEY R.S., 2002. The effects of surgically-implanted dummy radio transmitters on the behaviour of wild Atlantic salmon smolts. Hydrobiologia, 483, 231-237.

COWX I.G., LAMARQUE P., 1990. Fishing with electricity. Applications in freshwater Fisheries Management. Oxford: Fishing News Books Blackwell Scientific Publications Ed. 248 p.

DEROZIER C., 1989. L'anesthésie des poissons au phénoxy-2-éthanol. Thèse, Ecole Nationale Vétérinaire de Nantes, $56 \mathrm{p}$.

GILDERHUS P.A., MARKING L.L., 1987. Comparative efficacy of 16 anesthesic chemicals on rainbow trout. North American Journal of Fisheries Management, 7, 288-292.

GOSSET C., 1974. Cuve galvanonarcotique pour la mensuration des poissons. Bull. Fr. Pêche Piscic., 255, 72-76.

LAMARQUE P., 1967. Electrophysiology of fish subject to the action of a electric field. In « Fishing with electricity - Its applications to biology and management, pp. 65-92 ». V. R. Ed., FAO Fishings News books Ltd, Rome, pp. 276.

LUCAS M.C., 1989. Effects of implanted dummy transmitters on mortality, growth, and tissue reaction in rainbow trout, Salmo gairdneri Richardson. J. Fish Biol, 35, 577587.

MADDEN J.A., HOUSTON A.H., 1976. Use of electroanaesthesia with freshwater teleosts : some physiological consequences in the rainbow trout, Salmo gairdneri Richardson. J. Fish Biol., 9, 457-462.

MARINE RESEARH INSTITUTE, 1999. Concerted Action «Improvement of Tagging Methods for Stock Assessment and Research in Fisheries" (CATAG), FAIR. CT.96.1394, Final Report May 1999 (DRAFT). En ligne. <http ://www.hafro.is/catag/ g-reference/contents_page.html>. Consulté le 21 janvier 2003.

MARTIN S.W., LONG J.A., PEARSONS T.N., 1995. Comparison of survival, gonad development, and growth between rainbow trout with and without surgically implanted dummy radio transmitters. North American Journal of Fisheries Management, 15, 494-498.

MARTY G.D., SUMMERFIELD R.C., 1986. Pathways and mechanisms for expulsions of surgically implanted dummy transmitters from channel catfish. Trans. Am. Fish. Soc., 115, 577-589.

MELLAS E.J., HAYNES J.M., 1985. Swimming performance and behavior of rainbow trout (Salmo gairdneri) and white perch (Morone americana) : effects of attaching telemetry transmitters. Can. J. Fish Aquat. Sci., 42, 488-493. 
MOORE A., RUSSEL I.C., POTTER E.C.E., 1990. The effects of intraperitoneally implanted dummy acoustic transmitters on the behaviour and physiology of juvenile Atlantic salmon (Salmo salar). J. Fish. Biol., 37, 713-721.

MUNDAY P.L., WILKSON S.K., 1997. Comparative efficacy of clove oil and other chemicals in anaesthetization of Pomacentrus amboinensis, a coral reef fish. J. Fish Biol., 51, 931-938.

OVIDIO M., 1999. Tactiques et stratégies individuelles d'utilisation spatio-temporelle de l'habitat et des ressources alimentaires chez la truite commune (Salmo trutta L.) : étude par radio-pistage dans l'Aisne et l'Ourthe. Thèse, Université de Liège, Faculté des Sciences, Institut de Zoologie, $196 \mathrm{p}$.

OVIDIO M., PHILIPART J.C., 2002. The impact of small physical obstacles on upstream movements of six species of fish. Hydrobiologia, 483, 55-69.

ROSS M.J., KLEINER C.F., 1982. Shielded-needle technique for surgically implanting radio-frequency transmitters in fish. Progressive Fish-Culturist, 44, 41-43.

SOKAL R.R., ROHLF F.J., 2001. Biometry. The principles and practice of statistics in biological research. Third edition. W. H. Freeman and Company, New York.

STEVEN W.M., LONG J.A., PEARSON T.N., 1995. Comparison of survival, gonad development and growth between rainbow trout with and without surgically implanted dummy radio transmitters. North American Journal of Fisheries Management, 15, 494-498.

SUMMERFELT R.C., MOSIER D., 1984. Transintestinal expulsion of surgically implanted dummy transmitters by channel catfish. Transactions of the American Fisheries Society, 113, 760-766.

SWANBERG T.R., GEIST D.R., 1997. Effects of intraperitoneal transmitters on the social interaction of rainbow trout. North American Journal of Fisheries Management, 17, 178-181.

THOREAU X., BARAS E., 1996. Anesthesia and surgery procedures for implanting telemetry transmitters into the body cavity of tilapia Oreochromis aureus. Underwater Biotelemetry, E. BARAS, J. C. PHILIPPART eds., pp. 13-22.

VETOFISH, 2002. Généralités sur l'anesthésie des poissons. En ligne. <http://www. vetofish.com/article.php?storyid=35>. Consulté le 14 janvier 2003.

WAGNER G.N., STEVENS E.D., 1999. Effects of different surgical techniques : suture material and location of incision site on the behaviour of rainbow trout (Onchorynchus mykiss). Mar. Fresh. Physiol., 33, 103-114. 